\title{
Sustentabilidade no uso medicinal de jatobá (Hymenaea courbaril L. e H. stigonocarpa Mart. Ex Hayne) e a efetividade da Estação Ecológica Serra das Araras na manutenção destas espécies
}

Objetivou-se com este trabalho verificar se as espécies Hymenaea courbaril L. e H. stigonocarpa Mart. Ex Hayne localizadas próximas a Estação Ecológica Serra das Araras (EESA) estão sendo utilizadas de maneira sustentável, e se há uso destas espécies dentro das delimitações da EESA. Foram coletadas informações de 10 indivíduos das espécies Hymenaea courbaril L. e H. stigonocarpa Mart. Ex Hayne dentro das delimitações da EESA, e de 10 localizadas próximas a residências e à estrada de acesso da estação, sendo que a mais distante se situava a $10 \mathrm{~km}$ em direção ao município de Porto Estrela. Os dados coletados foram à altura do fuste, diâmetro a altura do peito, circunferência a altura do peito, comprimento da maior injúria, largura da maior injúria, número de injúrias e se estas já estavam cicatrizadas ou não. Verificou-se que houve diferença para as variáveis analisadas dentro e fora da EESA, demonstrando que a estação ecológica está cumprindo com o seu papel de conservação, mas também fora da mesma, o uso desta espécie parece não estar sendo realizado de maneira sustentável. Adicionalmente, recomenda-se um trabalho de educação ambiental com a população local, para que o uso deste recurso seja realizado de maneira sustentável, e ainda que se defina qual o número e tamanho das injúrias que garantam a regeneração da árvore para que se promova efetivamente o uso e a conservação destas espécies fora da Estação Ecológica Serra das Araras.

\section{Sustainable medicinal use of Jatobá (Hymenaea courbaril L. e H. stigonocarpa Mart. Ex Hayne) and the effectiveness of the Serra das Araras Ecological Station in the maintenance of these species}

We aimed with this research to verify if the species Hymenaea courbaril L. and H. stigonocarpa Mart. Ex Hayne located near the Serra das Araras Ecological Station (EESA) are being used in a sustainable way, and if these species are used on the EESA. Were collected information of 10 individuals of the species Hymenaea courbaril L. and H. stigonocarpa Mart. Ex Hayne on the EESA boundaries, and 10 located close to residences and the access road of the station, the most distant one being located $10 \mathrm{~km}$ towards the municipality of Porto Estrela. The data collected were the height of the shaft, diameter of breast height, circumference of breast height, length of major injury, width of major injury, number of injuries and whether or not they were healed. It was verified that there was a difference for the variables analyzed inside and outside the EESA, demonstrating that the ecological station is fulfilling its conservation role, but also outside it, the use of this species seems not to be carried out in a sustainable way. In addition, environmental education is recommended with the local population, so that the use of this resource is carried out in a sustainable way, and even if it is defined the number and size of the injuries that guarantee the regeneration of the tree so that it is promoted effectively the use and conservation of these species outside of the Serra das Araras Ecological Station.

Keywords: Environmental education; Pharmacognosy; Phytotherapeutics; Use and conservation.

Topic: Uso Sustentável da Biodiversidade

Reviewed anonymously in the process of blind peer.
Received: 02/10/2020

Approved: $21 / 11 / 2020$
Rafael Kill Silveira (iD

Universidade do Estado de Mato Grosso, Brasil

http://lattes.cnpq.br/2930558925471338

http://orcid.org/0000-0003-1902-8575

kill.silveira@unemat.br

Guilherme Augusto Nogueira Borges (ic)

Universidade do Estado de Mato Grosso, Brasil http://lattes.cnpq.br/2770247199984452

http://orcid.org/0000-0002-0313-7550

ganborgess@gmail.com

Maria Antonia Carniello ic

Universidade do Estado de Mato Grosso, Brasil

http://lattes.cnpq.br/6659152924492446

http://orcid.org/0000-0002-7474-4227

carniello@unemat.br

\author{
Manoel dos Santos Filho (D) \\ Universidade do Estado de Mato Grosso, Brasil \\ http://lattes.cnpq.br/9309680477779428 \\ http://orcid.org/0000-0002-9784-7114 \\ msantosfilho@gmail.com \\ Lisiane Vieira Paludetti \\ Universidade do Estado de Mato Grosso, Brasi \\ http://lattes.cnpq.br/3710224112810482 \\ http://orcid.org/0000-0003-1448-6470 \\ lisianevieira1@hotmail.com \\ Donei José da Silva (ic) \\ Universidade do Estado de Mato Grosso, Brasil \\ http://lattes.cnpq.br/5447213851327253 \\ http://orcid.org/0000-0002-6189-9756 \\ dioneijs@gmail.com
}

\section{Referencing this:}

SILVEIRA, R. K.; BORGES, G. A. N.; CARNIELLO, M. A.; SANTOS FILHO, M.; PALUDETTI, L. V.; SILVA, D. J.. Sustentabilidade no uso medicinal de jatobá (Hymenaea courbaril L. e H. stigonocarpa Mart. Ex Hayne) e a efetividade da Estação Ecológica Serra das Araras na manutenção destas espécies. Revista Ibero Americana de Ciências Ambientais, v.11, n.6, p.202-208, 2020. DOI: http://doi.org/10.6008/CBPC2179$\underline{6858.2020 .006 .0017}$ 


\section{INTRODUÇÃO}

Apesar do avanço na medicina alopática no mundo, existem algumas dificuldades para sua utilização pelas populações carentes, que vão desde o acesso aos centros de atendimento hospitalares, à obtenção de exames e medicamentos (VEIGA JUNIOR et al., 2005). De acordo com o Ministério da Saúde (2006), a Organização Mundial da Saúde (OMS) reconhece que nos países em desenvolvimento 80\% da população depende de práticas tradicionais nos cuidados básicos de saúde, e que $85 \%$ destes recorrem a plantas para realizar tal procedimento. Sendo está muitas vezes a única forma de tratamento para populações de baixo poder aquisitivo (PANIS, 2008).

Segundo Coan et al. (2013), o uso de plantas medicinais no Brasil se deve principalmente à riqueza e variedade de espécies da flora nativa. Associado a isto temos também a tradição no uso destes produtos, pois segundo Lahr (1997) as presenças dos ameríndios datam entre 11 e 50 mil anos atrás, ou seja, um longo período de experimentação e observação dos efeitos das plantas sobre a saúde. Dentre inúmeras plantas utilizadas com finalidade farmacológicas, podemos destacar o uso das espécies Hymenaea courbaril L. e $H$. stigonocarpa Mart. Ex Hayne, conhecidas popularmente por Jatobá (dentre outros nomes populares), estas são plantas pertencentes ao grupo das angiospermas e família fabaceae, tendo distribuição em todas as regiões do Brasil e nos domínios fitogeográficos da Amazônia, Caatinga, Cerrado, Mata Atlântica e Pantanal.

Existem relatos do uso tradicional do Jatobá para inúmeras enfermidades, destacando-se sua utilização para a bronquite, tosse, gripe, inflamações, pneumonia, etc. (CHAVES et al., 2012; ALCANTARA et al., 2015; ALMEIDA et al., 2002; FRANCO et al., 2006; MORAES et al., 2011). Havendo comprovação científica que estas espécies contêm terpenos e compostos fenólicos (dentre outras substâncias) com comprovada atividade restauradora da saúde (MARSAIOLI, 1975; SALES et al., 2014; GONÇALVES et al., 2013; PEREIRA et al., 2007, GONÇALVES et al., 2005; JAYAPRAKASAM et al., 2007).

Para as espécies utilizadas com fins medicinais é importante ressaltar que as mesmas estão suscetíveis ao uso não sustentável, principalmente aquelas em que as técnicas de coleta são altamente agressivas para a planta, como é o caso do jatobá, em que a parte utilizada é a casca do caule (ALMEIDA et al., 2002). Alguns autores têm sugerido que o simples fato das populações tradicionais locais utilizarem algumas espécies vegetais garante a sua preservação (SILVA et al., 2018; PEDROZO et al., 2011; OLIVEIRA et al., 2015). No entanto, até mesmo para as comunidades tradicionais o uso não garante a conservação (COAN et al., 2013). Uma das formas utilizadas para a manutenção de espécies vegetais e animais é a criação das Estações ecológicas, para que neste local não se possa fazer o uso de qualquer recurso e com isso garantir sua preservação.

Neste contexto objetivou-se com este trabalho verificar se as espécies Hymenaea courbaril L. e $H$. stigonocarpa Mart. Ex Hayne localizadas próximas a Estação Ecológica Serra das Araras (EESA) estão sendo utilizadas de maneira sustentável, e se há uso destas espécies dentro das delimitações da EESA. 


\section{MATERIAIS E MÉTODOS}

Os dados foram coletados na Estação Ecológica Serra das Araras (EESA), situada entre os municípios de Porto Estrela, Cáceres e Barra do Bugres, MT, entre as latitudes $15^{\circ} 27^{\prime}$ e $15^{\circ} 48^{\prime}$ S e longitudes $57^{\circ} 03^{\prime}$ e $57^{\circ} 19^{\prime}$ W, com altitudes variando entre 300 e 800 m (SILVA et al., 1988). De acordo com Gonçalves et al. (2004), o clima é tropical quente subsumido (Aw) e precipitação anual em torno de $1.400 \mathrm{~mm}$. A média das temperaturas mínimas e máximas é de $20^{\circ} \mathrm{C}$ e $32^{\circ} \mathrm{C}$, respectivamente, com duas estações bem definidas chuvosa (novembro a abril) e seca (demais meses do ano). Segundo Valadão (2012), a EESA é composta pelas fitofisionomias mata semidecidual, mata ciliar, cerradão, cerrado sentido restrito e Parque cerrado.

Aleatoriamente foram coletadas informações de 10 indivíduos das espécies Hymenaea courbaril L. e H. stigonocarpa Mart. Ex Hayne dentro das delimitações da EESA, e de 10 localizadas próximas a residências e à estrada de acesso da estação, sendo que a mais distante se situava a $10 \mathrm{~km}$ em direção ao município de Porto Estrela. Os dados coletados foram à altura do fuste, diâmetro a altura do peito, circunferência a altura do peito, comprimento da maior injúria, largura da maior injúria, número de injúrias observadas em cada planta, e ainda foram contadas o número de injúrias e se estas estavam cicatrizadas ou não. Para mensurar a Altura do fuste foi utilizada trena a laser, e para as medidas de diâmetro a altura do peito, circunferência a altura do peito, comprimento da maior injúria, largura da maior injúria foi utilizado fita métrica.

Foi mensurado também a área das injúrias, para tal foi utilizado um saco plástico transparente colocado sobre a injúria, e com uma caneta marcador permanente foi desenhado o contorno da injúria (decalque), posteriormente foi calculado a área da injúria com o uso de papel quadriculado (quadros de $1 \mathrm{~cm}$ ) sob o plástico com o decalque da injúria e contado o número de quadros que estavam dentro do decalque. Para as injúrias muito grandes em que não foi possível fazer o decalque foram aferidas a medida da largura e comprimento, sendo posteriormente multiplicadas e encontrada a área, neste caso cálculo da área não fica exato, mas esta medida foi adotada para todos os indivíduos analisados. As medidas de maior comprimento e maior largura da injúria foram utilizadas para determinar se o uso é sustentável ou não, uma vez que uma injúria muito grande pode impossibilitar a árvore se recuperar da lesão, predispondo-a à morte.

Devido aos dados não apresentarem distribuição normal pelo teste de Shapiro-wilk, foi empregado o teste de Mann-whitney para as variáveis comprimento da maior injúria, largura da maior injúria, número de injúrias observadas em cada planta. Para a verificar se o número de árvores com injúrias cicatrizadas foi maior dentro ou fora da EESA utilizou-se o teste de Qui-quadrado. Todas as análises estatísticas foram realizadas utilizando o software $\mathrm{R}$ versão 3.4.4.

\section{RESULTADOS E DISCUSSÃO}

Ao aplicar o teste de Qui-quadrado com a finalidade de verificar se os números de árvores estavam com suas injúrias cicatrizadas ou não, foi possível observar que fora da EESA o número de plantas com injúrias não cicatrizadas é muito maior do que na EESA (Figura 1). 


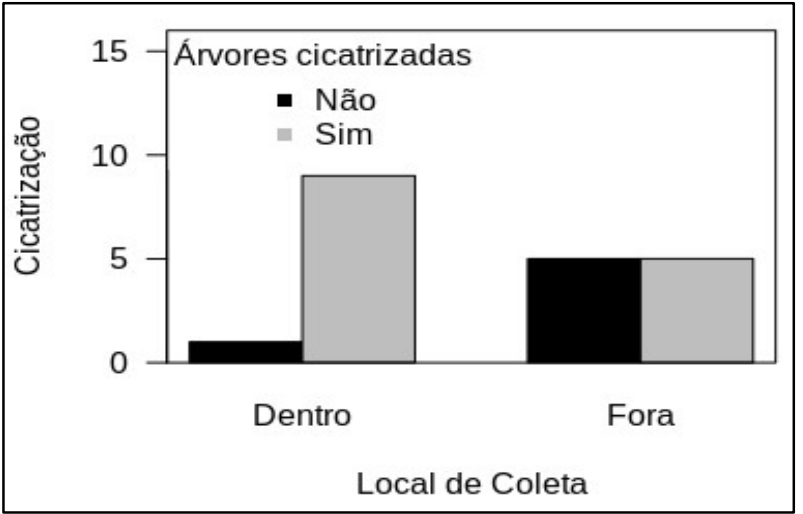

Figura 1: Número de indivíduos com injúrias cicatrizadas ou não dentro e fora da EESA.

A variável cicatrização se refere indiretamente ao tempo entre a retirada do súber e a nossa visualização, desta forma esta variável é uma medida que avalia de utilização ou não desta espécie como recurso, mas também é uma medida de temporalidade do uso, demonstrando assim, que dentro da estação houve uso destas espécies, no entanto sua utilização se dava de maneira menos intensa (apenas um indivíduo). E fora da estação há uso recente e intenso, no entanto, com o uso desta variável não é possível definir se o uso está sendo realizado de maneira sustentável, pois devido à característica temporal não poderemos afirmar se as injúrias irão cicatrizar ou não.

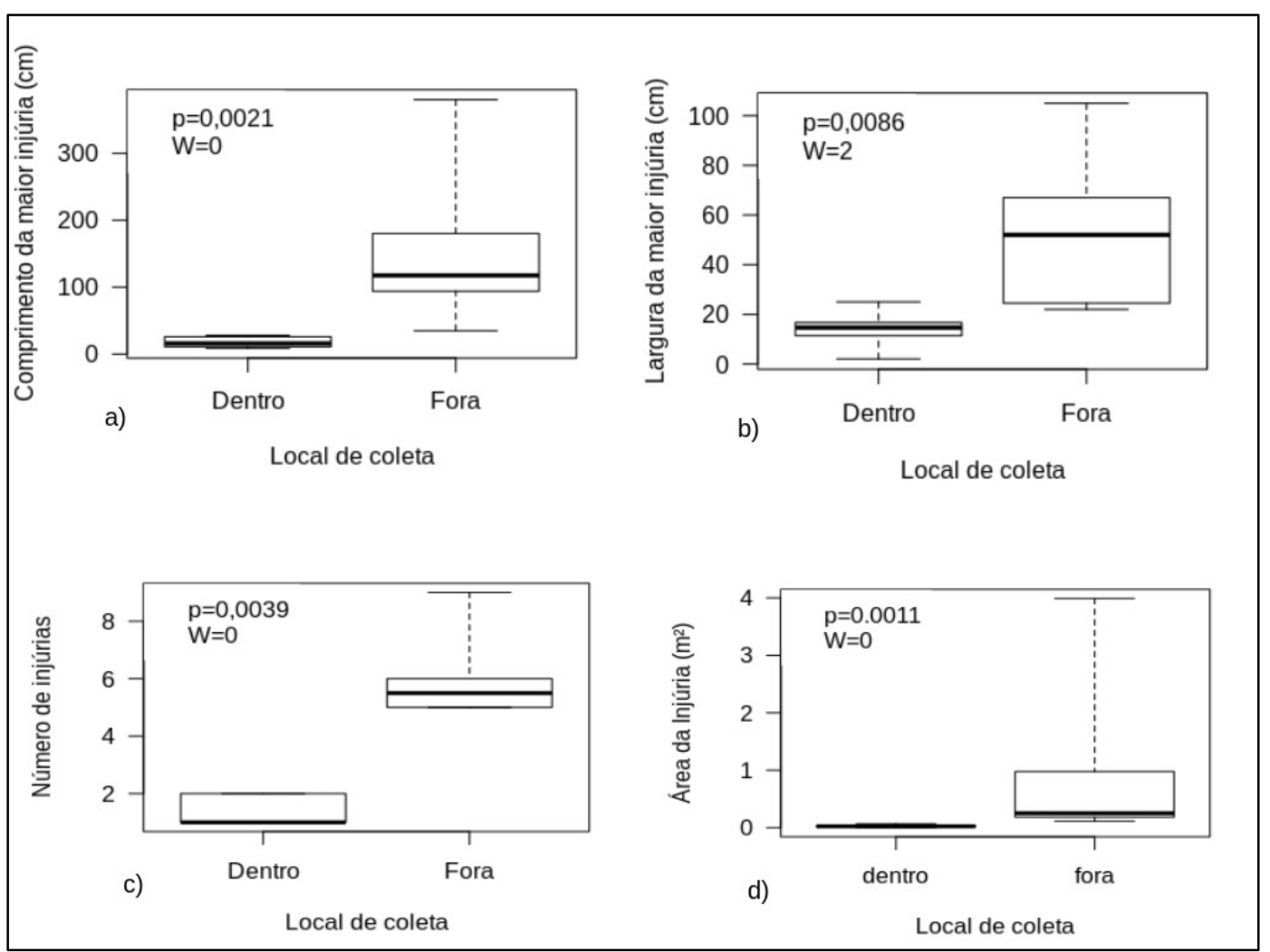

Figura 2: a) Comprimento da maior injúria, b) Largura da maior injúria, c) Número de injúrias e d) Área da injúria, dentro e fora da EESA.

Ao aplicar o teste de Mann-whitney para a variável comprimento da maior injúria, largura da maior injúria, número de injúrias e área da injúria de cada indivíduo foi possível observar que houve diferença para as variáveis analisadas dentro e fora da EESA (Figura 2). 
Isto demonstra que a estação ecológica está cumprindo com o seu papel de conservação, mas também fora da mesma, o uso desta espécie parece não estar sendo realizado de maneira sustentável. Todas as árvores analisadas estavam vivas e para ambos os ambientes, no entanto algumas árvores localizadas fora da estação ecológica provavelmente não sobreviverão por muito tempo devido ao tamanho e número de suas lesões.

Segundo o ICMBIO a Estação Ecológica Serra das Araras, unidade de conservação federal da categoria de proteção integral foi criada pelo decreto 87.222 , de 31 de maio de 1982, no entanto, as árvores de jatobá ainda apresentam sinais de uso pretérito, mas os números de lesões são menores assim como o tamanho quando comparado a aquelas árvores localizadas fora da EESA. Isto nos leva a sugerir que no passado o uso se dava de maneira mais sustentável. Na tentativa de compreender quais os motivos pelos quais o manejo era mais sustentável no passado, podemos no questionar que talvez as populações daquela época tinham maior consciência ambiental? Ou será que o número de árvores era muito maior, tendo assim maior disponibilidade de recursos e como consequência o uso se dava de maneira mais sustentável?.

Possivelmente fatores como o desmatamento para uso destas áreas como pastagem e/ou agricultura, o uso do jatobazeiro para construções como pontes, casas, currais, cercas dentre outros usos, reduziu significativamente o número de indivíduos nas áreas fora da EESA, além de que, com o desmatamento algumas poucas árvores desta espécie que restaram estão mais visíveis para aqueles que a procuram como fonte medicamentosa. Outro fator que pode estar associado a maior pressão de uso desta espécie é o aumento da população humana na área estudada ao longo dos anos.

Uma medida eficiente para tentar amenizar o uso excessivo desta espécie seria a educação ambiental da população local, de maneira a lhes orientar da futura perda total deste recurso. Sendo está a recomendação de Coan et al. (2013), quando trabalharam com uma comunidade indígena no RS, e perceberam necessidade de orientação aos indígenas para o manejo sustentável das espécies extraídas, principalmente para aquelas espécies em que se utilizam a casca do tronco. No entanto, somente isto não seria o suficiente pois os agentes locais também teriam que desempenhar papel de fiscalizadores quanto ao uso desta planta, pois existem comerciantes que não residem no local e que utilizam este recurso para venda como medicamento em outras localidades. Seria pertinente empregar a recomendação de Gonçalves et al. (2013), que indicam o reflorestamento com estas espécies em áreas degradadas, e isto adicionalmente contribuiria com a geração de renda local.

Estes fatos corroboram com Almeida et al. (2002), pois ao levantarem informações sobre o uso medicinal de plantas e discutirem sobre as implicações do uso e extrativismo das espécies, observaram que muitas plantas comercializadas, que gozam de maior popularidade, estão vulneráveis pela perseguição sistemática dos comerciantes. Ações devem ser tomadas pois a utilização inadequada deste recurso poderá se intensificar, uma vez que de acordo com França et al. (2008), nas últimas décadas o consumo de plantas medicinais aumentou em todo o mundo. 
No caso do Jatobá as mesmas estarão aptas ao uso medicinal após possuírem um tamanho relativamente grande, logo existe ainda outros agravantes inerentes a esta espécie, destacando-se o crescimento lento e baixa taxa de germinação (SANTOS et al., 2008; PIEREZAN et al., 2012; MELO et al., 2004), associado a um problema de dispersão das sementes, que só é realizado efetivamente por Cutias (GORCHOV et al., 2004), e esta espécie não habita em áreas abertas (CID, 2011). Desta forma em caso de redução drástica da população de Jatobás o processo de replantio terá que ser realizado pelo homem, atentando-se para uma outra característica importante que na fase jovem se desenvolve melhor sob sombreamento (PIEREZAN et al., 2012), e as árvores encontradas fora da EESA estavam em pleno sol.

Não há como afirmar com toda certeza se o uso desta espécie está sendo realizado de maneira sustentável ou não, pois não existe metodologia que defina qual o número, largura, comprimento e área das injúrias que as árvores possam se recuperar em sua totalidade e não sofrer um processo de senescência e morte. Não há ainda na literatura trabalhos que definam qual a área máxima que pode ser retirada do súber de maneira que a planta se recupere, para que seu uso possa se dar realmente de maneira sustentável.

\section{CONCLUSÕES}

Houve diferença para as variáveis analisadas dentro e fora da EESA demonstrando que a estação ecológica está cumprindo com o seu papel de conservação, mas também fora da mesma, o uso desta espécie parece não estar sendo realizado de maneira sustentável. Adicionalmente recomenda-se um trabalho de educação ambiental com a população local para que o uso deste recurso seja realizado de maneira sustentável, e ainda que se defina qual o número e tamanho das injúrias que garantam a regeneração da árvore para que se promova efetivamente o uso e conservação destas espécies fora da Estação Ecológica Serra das Araras.

\section{REFERÊNCIAS}

ALCANTARA, R. G. L.; JOAQUIM, R. H. V. T.; SAMPAIO, S. F.. Plantas medicinais: o conhecimento e uso popular. Revista APS, v.18, n.4, p.470-482, 2015

ALMEIDA, C. F. C. B. R.; ALBUQUERQUE, U. P.. Uso e conservação de plantas e animais medicinais no Estado de Pernambuco (Nordeste do Brasil): um estudo de caso. Interciência, v.27, n.6, p.276-285, 2002.

BRASIL. Ministério da Saúde. Política Nacional de Plantas Medicinais e Fitoterápicos. Dispõe sobre as diretrizes da implantação de plantas medicinais e fitoterápicos no SUS. Brasília: Ministério da Saúde, 2006.

CHAVES, E. M. F.; BARROS, R. F. M.. Diversidade e uso de recursos medicinais do carrasco na APA da Serra da Ibiapaba, Piauí, Nordeste do Brasil. Revista Brasileira de Plantas Medicinais, v.14, n.3, p.476-486, 2012.

CID, B.. Reintrodução da cutia-vermelha (Dasyprocta leporina) no Parque Nacional da Tijuca (Rio de Janeiro, RJ): avaliação dos procedimentos, determinação do sucesso em curto prazo e caracterização dos padrões espaciais.
Dissertação (Mestrado em Ecologia) -Universidade Federal do Rio de Janeiro, Rio de Janeiro, 2011.

COAN, C. M.; MATIAS, T.. Utilização das plantas medicinais pela comunidade indígena de Ventarra Alta/RS. Revista de Educação do IDEAU, v.8, n.18, 2013.

FRANÇA, I.; SOUZA, J.; BAPTISTA, R.; BRITTO, V.. Medicina popular: benefícios e malefícios das plantas medicinais. Revista Brasileira de Enfermagem, v.61, n.2, p.201-208, 2008.

FRANCO, E. A. P.; BARROS, R. F. M.. Uso e diversidade de plantas medicinais no Quilombo Olho D'água dos Pires, Esperantina, Piauí. Revista Brasileira de Plantas Medicinais, v.8, n.3, p.78-88, 2006.

GONÇALVES, A. L.; ALVES FILHO, A.. Estudo comparativo da atividade antimicrobiana de extratos de algumas árvores nativas. Arquivos do Instituto de Biologia, n.72, p.353-358, 2005. 
GONÇALVES, A. L.; ALVES FILHO, A.; MENEZES, H.. Árvores medicinais nativas com potencial para extrativismo autossustentável-atividade antimicrobiana. Global Science and Technology, v.06, n.02, p.114-120, 2013.

GORCHOV, D. L.; PALMEIRIM, J. M.; JARAMILLO, M.; ASCORRA, C. F.. Dispersal of seeds of Hymenaea courbaril (Fabaceae) in a logged rain forest in the Peruvian Amazonian. Acta Amazônica, v.34, n.2, p.251-259, 2004.

JAYAPRAKASAM, B.; ALEXANDER-LINDO, R. L.; WITT, D. L.; NAIR, M. G.. Terpenoids from Stinking toe (Hymenaea courbaril) fruits with cyclooxygenase and lipid peroxidation inhibitory activities. Food Chemistry, v.105, p.485-490, 2007.

LAHR, M. M.. Origem dos ameríndios no contexto da evolução dos povos mongoloides. Revista USP, n.34, p.7081, 1997.

MARSAIOLI, A. J.. Diterpenes in the bark of Hymenaea courbaril. Phytochemistry, v.14, p.1882-1883, 1975.

MELO, M. G. G.; MENDONÇA, M. S.; MENDES, A. M. S.. Análise morfológica de sementes, germinação de plântulas de jatobá (Hymenaea intermedia Duckevar. Adenotricha (Ducke) Lee \& Lang.) (Leguminosae-caesalpinioideae). Acta Amazônica, v.34, n.1, p.9-19, 2004.

MORAES, L. G.; ALONSO; A. M; OLIVEIRA FILHO, E. C.. Plantas medicinais no tratamento do câncer: uma breve revisão de literatura. Universitas: Ciências da Saúde, v.9, n.1, p.77-99, 2011.

OLIVEIRA, V.; SANTOS, E. M. M.; MESQUITA, E. V. A.. Prescrição, preparo e manipulação de plantas medicinais. Saber Científico, v.4, n.1, p.40-45, 2015.

PANIS, C.. Plantas do gênero Bacharis em fitote-rapia. Uso popular x Comprovação científica. Infarma, v.20, p.30-34, 2008.

PEDROZO, E. A.; SILVA, T. N.; SATO, S. A. S.; OLIVEIRA, N. D. A.. Produtos Florestais Não Madeiráveis (PFNMS): as Filières do Açaí e da Castanha da Amazônia. Revista de

Administração e Negócios da Amazônia, v.3, n.2, 2011.

PEREIRA, C. K. B.; RODRIGUES, F. F. G.; MOTA, M. L.; SOUSA, E. O.; LEITE, G. O.; BARROS, A. R. C.; LEMOS, T. L. G.; COSTA, J. G. M.. Composição química, atividade antimicrobiana e toxicidade do óleo essencial de Hymenaea courbaril (Jatobá). In: REUNIÃO ANUAL DA SOCIEDADE BRASILEIRA DE QUÍMICA, 30. Anais. SBQ, 2007.

PIEREZAN, L.; SCALON, S. P. Q.; PEREIRA, Z. V.. Emergência de plântulas e crescimento de mudas de jatobácom uso de bioestimulante e sombreamento, Cerne, v.18, n.1, p.127133, 2012.

SALES, G. W. P.; BATISTA, A. H. M.; ROCHA, L. Q.; NOGUEIRA, N. A. P.. Efeito antimicrobiano e modulador do óleo essencial extraído da casca de frutos da Hymenaea courbaril L. Revista de Ciências Farmacêuticas Básica e Aplicada, v.35, n.4, p.708-715, 2014.

SANTOS, J. Z. L.; RESENDE, Á. V.; FURTINI NETO, A. E.; CORTE, E. F.. Crescimento, acúmulo de fósforo e frações fosfatadas em mudas de sete espécies arbóreas nativas. Revista Árvore, v.32, n.5, p.799-807, 2008.

SILVA, J. M. C.; ONIKI, Y.. Lista preliminar da avifauna da Estação Ecológica Serra das Araras, Mato Grosso, Brasil. Boletim do Museu Paraense Emílio Goeldi, 1988.

SILVA, M. R.; LAMARCA, E. V.. registros etnobotânicos e potenciais medicinais e econômicos do jatobá (Hymenaea courbaril), Revista Ibirapuera, n.15, p.8-12, 2018.

VALADÃO, R. M.. As aves da Estação Ecológica Serra das Araras, Mato Grosso, Brasil. Biota Neotropical, v.12, n.3, 2012.

VEIGA JUNIOR, V. F.; PINTO, A. C.; MACIEL, M. A. M.. Plantas medicinais: Cura segura?. Química Nova, v.28, n.3, p.519$528,2005$.

A CBPC - Companhia Brasileira de Produção Científica (CNPJ: 11.221.422/0001-03) detém os direitos materiais desta publicação. Os direitos referem-se à publicação do trabalho em qualquer parte do mundo, incluindo os direitos às renovações, expansões e disseminações da contribuição, bem como outros direitos subsidiários. Todos os trabalhos publicados eletronicamente poderão posteriormente ser publicados em coletâneas impressas sob coordenação da Sustenere Publishing, da Companhia Brasileira de Produção Científica e seus parceiros autorizados. Os (as) autores (as) preservam os direitos autorais, mas não têm permissão para a publicação da contribuição em outro meio, impresso ou digital, em português ou em tradução. 